\title{
Individual fairness in Harsanyi's utilitarianism: operationalizing all-inclusive utility
}

\author{
Stefan T. Trautmann
}

Published online: 17 April 2008

(C) The Author(s) 2008

\begin{abstract}
Fairness can be incorporated into Harsanyi's utilitarianism through all-inclusive utility. This retains the normative assumptions of expected utility and Pareto-efficiency, and relates fairness to individual preferences. It makes utilitarianism unfalsifiable, however, if agents' all-inclusive utilities are not explicitly specified. This note proposes a two-stage model to make utilitarian welfare analysis falsifiable by specifying all-inclusive utilities explicitly through models of individual fairness preferences. The approach is applied to include fairness in widely discussed allocation examples.
\end{abstract}

Keywords Utilitarianism - Outcome fairness - Process fairness · All-inclusive utility

\section{Introduction}

Harsanyi (1955) derived the utilitarian social welfare function assuming expected utility for individuals and the social planner, and assuming the Pareto-principle. The result has been criticized because the linear form of the social welfare function, allegedly, precludes considerations of fairness by the social planner. Fairness can be incorporated into Harsanyi's framework through a description of social allocations that includes, apart from the individuals' personal situations, all interpersonal comparisons (Harsanyi 1955; Broome 1991; Binmore 1994; Karni 1996). Individual utilities over these complete descriptions of allocations are all-inclusive.

S. T. Trautmann $(\varangle)$

Econometric Institute, Erasmus University Rotterdam, P.O. Box 1738, 3000 DR Rotterdam,

The Netherlands

e-mail: trautmann@few.eur.nl 
The all-inclusive utility approach allows the social welfare function to be based on the normatively convincing assumptions of expected utility and Pareto-efficiency, while considering agents' individual attitudes towards fairness. Fairness has a clear interpretation in terms of individual preferences and need not be considered separately by the social planner (Fischer and Torgler 2006; van Winden 2007). Without explicit specification of the individual fairness preferences, however, the approach deprives Harsanyi's theory from predictive power and makes it unfalsifiable. Utility becomes context-dependent and every preference of the social planner between allocations can be accommodated by including all fairness issues in the agents' utilities. This has usually been the stalemate position in which debates about utilitarianism end, with either accepting the unsatisfactory lack of fairness considerations or accepting a loss of operationality.

This article offers a way out of the stalemate based on models of individual fairness preferences. The predictive power and falsifiability of utilitarianism with all-inclusive utility can be maintained by explicitly specifying testable and context-independent fairness preferences for individuals. A model is introduced that applies Sugden's (2000) two-stage procedure for game theory to operationalize fairness in all- inclusive utility and give empirical meaning to fairness in Harsanyi's utilitarianism. At the first stage, agents evaluate risky outcomes (health states, wealth levels) by self-interested von Neumann-Morgenstern (vNM) utilities that evaluate risky options without social comparison. No fairness considerations enter at this stage. At the second stage, the self-interested vNM utilities are then taken as inputs in models of individual fairness (Fehr and Schmidt 1999; Trautmann 2007) to obtain all-inclusive vNM utilities. These models provide operational functional forms for fairness preferences and successfully explain empirical data. They can numerically be assessed for individual agents from observable choices.

The two-stage approach is applied to well-known examples by Diamond (1967) and Broome (1991) which criticize utilitarianism on fairness grounds. Distinguishing between self-interested and all-inclusive vNM utilities allows us to derive convincing empirical predictions in these examples under utilitarianism.

The article is organized as follows. The next section discusses fairness-based criticisms of utilitarianism and all-inclusive utility. Section 3 introduces the two-stage model to operationalize fairness and obtain meaningful all-inclusive utilities. Section 4 applies the approach to analyze Diamond's and Broome's examples with individual fairness preferences. Section 5 discusses the results and the last section concludes.

\section{Utilitarianism, fairness, and all-inclusive utility}

Harsanyi (1955) used cardinal utility obtained from choices between risky allocations to derive a social welfare function. He assumed that the social planner and the individual agents use expected utility to evaluate risky prospects over outcomes, and that the Pareto-principle holds. The latter requires that indifference between two prospects for each individual imply indifference from the social standpoint. Harsanyi showed that these three assumptions imply a social welfare function $\mathrm{W}$ of the utilitarian form, $W=\sum_{i} U_{i}$, where the $U_{i}$ 's are the individual agents' vNM utilities over outcomes. 
Fig. 1 Random allocation of utilities in Diamond's example

Fig. 2 Random allocation of utilities in Broome's example
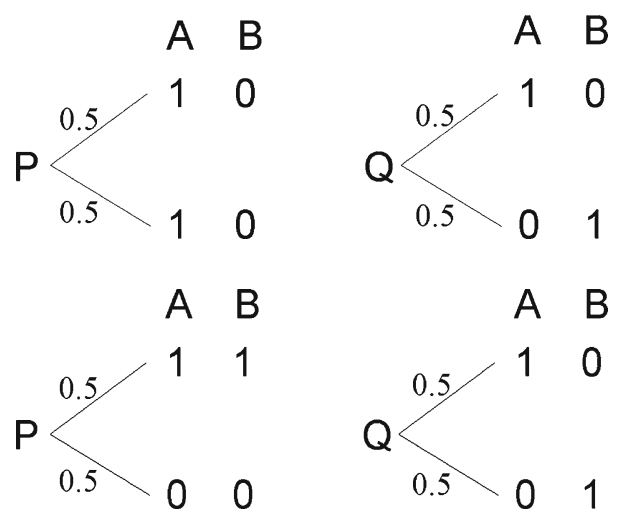

\subsection{Fairness-based criticisms of utilitarianism}

Harsanyi's result has been criticized because of the absence of fairness considerations in the evaluation of the utilitarian social planner. Consider the following two examples in which the utilitarian prediction of which random allocation should be preferred by society has been argued not to be convincing.

Example 1 (Diamond 1967) Consider the two random allocations $\mathrm{P}$ and $\mathrm{Q}$ of utilities for agents A and B in Fig. 1.

Under utilitarianism the planner is indifferent between procedures $\mathrm{P}$ and $\mathrm{Q}$ because both imply an expected social welfare of 1 . Indifference can be unconvincing because with procedure $\mathrm{P}$ agent $\mathrm{B}$ will receive nothing while $\mathrm{A}$ receives positive utility for sure. With procedure Q both agents have a fair chance of the same utility.

Example 2 (Broome 1991, p. 185) Consider the allocations in Fig. 2.

Under utilitarianism the planner is indifferent between procedures $\mathrm{P}$ and $\mathrm{Q}$ because both imply an expected welfare of 1 . Indifference can be unconvincing because allocation procedure $\mathrm{P}$ always leads to equality in terms of utilities, while $\mathrm{Q}$ always leads to inequality.

In Diamond's example utilitarianism violates process fairness, and in Broome's example it violates outcome fairness.

\subsection{Non-utilitarian social welfare versus all-inclusive utility}

In order to incorporate fairness in Harsanyi's framework, two approaches can be followed. First, the assumptions of expected utility and Pareto-efficiency can be weakened and a non-utilitarian social welfare function can be derived that accounts for fairness preferences of the social planner (Epstein and Segal 1992; Kelsey 1994; Wakker and Zank 1999; Grant etal. 2006; Zank 2007). However, it is not obvious that a social welfare function should be based on the assumption of non-expected utility evaluation of risky prospects by the social planner. Further, it is not clear whose 
fairness preferences are represented by this social evaluation if no agent in the society individually cares about fairness (van Winden 2007).

Second, it can be assumed that the description of the social allocations does not only include the individual agents' personal outcomes, but also all possible interpersonal comparisons (Harsanyi 1955; Luce and Raiffa 1957; Broome 1991; Binmore 1994; Karni 1996). The agents' vNM utilities of a social allocation, which are used in the social evaluation of the allocation, are all-inclusive.

The second approach seems normatively more convincing because it retains expected utility and Pareto-efficiency, and derives fairness from individual preferences (Karni 1996; Karni and Safra 2002). In particular, the individual fairness preferences may differ across agents. All-inclusive utility, however, also suffers from problems if taken in full generality. When observing an empirical violation of utilitarianism, it can always be argued that a refined description of outcomes that explicitly incorporates fairness can accommodate the empirically observed social preference. Thus, utilitarianism is deprived from predictive power and becomes unfalsifiable, as illustrated by the following example.

Example 3 Consider again Example 2 and interpret the entries as all-inclusive vNM utilities. The social planner is indifferent between both procedures under utilitarianism. Now consider a new decision for the planner that is identical to the one in Example 2, except that probabilities under procedure $Q$ are 0.25 for the upper branch and 0.75 for the lower branch. Assume that the all-inclusive utilities of the two agents in Fig. 2 are due to a purely selfish utility evaluation of outcomes, that is, agents do not care about fairness. Then the social choice with new procedure $\mathrm{Q}$ is given by the entries in Fig. 3. The planner is indifferent because the expected social welfare is equal to 1 for both procedures.

Alternatively, the all-inclusive utilities in Example 2 when taken completely general may include individual preferences for fairness. Assume for instance that the all-inclusive utilities depend on monetary payoffs and social comparisons of these payoffs. Assume that agents prefer more money to less and that they also prefer smaller interpersonal differences in expected payoffs because they value process fairness. The $U_{i}$ 's in Fig. 2 then are functions of agent i's own payoff and of the difference between her own expected payoff and the other agent's expected payoff. In this case, changing the probability distribution under procedure $\mathrm{Q}$ affects the agents' all-inclusive utilities, because the difference between the agents' expected payoffs changes. The social choice is defined by the entries in Fig. 4 for unknown all-inclusive utilities a, b, c, and $\mathrm{d}$, and indifference of the planner need not hold because $0.25(\mathrm{a}+\mathrm{b})+0.75(\mathrm{c}+\mathrm{d})$ need not be equal to 1 .

Fig. 3 Broome's example with
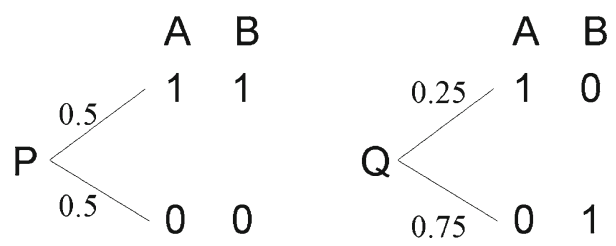
Fig. 4 Broome's example with different procedure Q: all-inclusive utilities when agents care about differences in expected payoffs

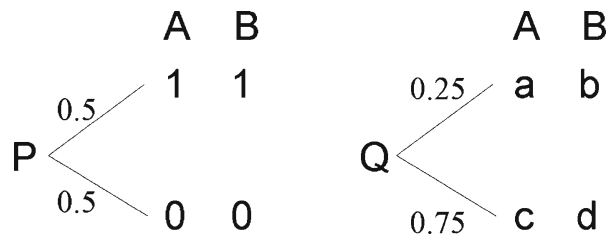

Assuming all-inclusive utilities, no prediction can be made about the society's decision in Example 3 based on the knowledge that the society is indifferent in Example 2. Indifference in Example 2 can be due to either selfishness or preference for fair expected outcomes, with very different implications for the social decision in Example 3. Without explicit and falsifiable assumptions concerning individual fairness preferences, the model with all-inclusive utility has no predictive power (Machina 1989; Broome 1991). It cannot be applied to evaluate social allocations.

\section{Operationalizing all-inclusive utility}

\subsection{The two-stage model}

A two-stage model that is based on Sugden's (2000) methodology to incorporate social interaction in game-theoretic analyses is proposed to operationalize fairness in all-inclusive utility. Let there be $\mathrm{N}$ agents with all-inclusive vNM utilities $U_{i}$ and a social planner with social welfare function W. $u_{i}$ denotes agent i's self-interested vNM utility and $z_{i}$ denotes her actual outcome. Welfare is evaluated by the following model:

$$
\begin{array}{ll}
W=\sum_{i}^{N} U_{i} & \text { (utilitarianism) } \\
U_{i}=f_{i}\left(u_{1}, \ldots, u_{N}\right) & \text { (second stage) } \\
u_{i}=g_{i}\left(z_{i}\right) & \text { (first stage) }
\end{array}
$$

At the first stage, the agents' individual outcomes (health states, wealth levels) are evaluated through the self-interested vNM utilities that evaluate risky options without social comparison. Agents consider only their personal situation, and no fairness considerations enter at this stage. At the second stage, these self-interested vNM utilities are then taken as inputs in models of individual fairness preferences $f_{i}(\cdot)$ to obtain all-inclusive vNM utilities for utilitarian welfare evaluation. An agent's all-inclusive utility may depend on both her own and all other agents' self-interested utilities.

The first-stage self-interested utilities are functions of possibly non-numerical outcomes. The second-stage all-inclusive utilities are functions of numerical selfinterested utilities. This allows for fairness comparisons also if outcomes are not numerical as with health states. Differences or expected values of self-interested utilities of health states can be calculated at the second stage to obtain the all-inclusive utilities. 
A tractable parametric model of individual fairness that can be used at the second stage has been proposed by Fehr and Schmidt (1999). They consider individual preference for outcome fairness, that is, agents are averse to unequal outcomes. Trautmann (2007) extends the model to individual preference for process fairness by assuming aversion to unequal expected outcomes. Notice that outcome fairness and process fairness refer to the allocation of self-interested utility in the application of these models in the two-stage model. We will see that specifying individual fairness preferences explicitly through parametric models at the second stage makes all-inclusive utility operational and quantitatively formalizes the discussion of fairness under utilitarianism.

\subsection{Models of individual fairness}

Let the $N$ agents face uncertainty about the allocation of self-interested utilities. Let $X_{i}$ denote the random variable from which agent i's self-interested utilities will be drawn and let $E\left[X_{i}\right]$ denote its expectation. Assuming that the agents dislike an unequal ex-post allocation of self-interested utilities we apply the following all-inclusive utility function, the Outcome Fehr-Schmidt Model (Fehr and Schmidt 1999), to account for fairness preferences:

$$
U_{i}\left(u_{1}, \ldots, u_{N}\right)=u_{i}-\frac{\alpha_{i}}{N-1} \sum_{j \neq i}^{N} \max \left\{u_{j}-u_{i}, 0\right\}-\frac{\beta_{i}}{N-1} \sum_{j \neq i}^{N} \max \left\{u_{i}-u_{j}, 0\right\}
$$

with $0 \leq \beta_{i}<1$ and $\beta_{i} \leq \alpha_{i}$. This utility function takes the agent's fairness preferences into account by reducing the all-inclusive utility in the case of unequal actual self-interested utilities, i.e. $u_{i} \neq u_{j}$. The utility reduction is larger when the inequality is disadvantageous $\left(u_{i}<u_{j}\right)$ than when it is advantageous $\left(u_{i}>u_{j}\right)$, because $\beta_{i} \leq \alpha_{i}$. From $\beta_{i}<1$ it follows that the agent's all-inclusive utility is always increasing in her own self-interested utility, and non-negativity of the parameters implies that there is no inequality seeking. The normalization of the fairness terms by $N-1$ ensures that the impact of fairness remains constant if the number of agents increases.

The outcome Fehr-Schmidt model provides an operational and tractable form to model preferences for outcome fairness and has been very successful in predicting empirical data. Evidence from experiments using random allocations has shown, however, that agents also care about whether procedures are fair from the ex-ante perspective (Cox and Deck 2005; Bolton etal. 2005). Using the basic form of the Fehr-Schmidt model, but assuming that agents care about fair processes, Trautmann (2007) proposed the following all-inclusive utility function, the Process Fehr-Schmidt Model, that accounts for preferences for fairness by considering differences in the expected self-interested utilities between the agents:

$$
\begin{aligned}
U_{i}\left(u_{i}, X_{1}, \ldots, X_{N}\right)= & u_{i}-\frac{\alpha_{i}}{N-1} \sum_{j \neq i}^{N} \max \left\{E\left[X_{j}\right]-E\left[X_{i}\right], 0\right\} \\
& -\frac{\beta_{i}}{N-1} \sum_{j \neq i}^{N} \max \left\{E\left[X_{i}\right]-E\left[X_{j}\right], 0\right\}
\end{aligned}
$$


with $0 \leq \beta_{i}<1$ and $\beta_{i} \leq \alpha_{i}$. This utility function takes the agent's fairness preferences into account by reducing the all-inclusive utility in the case of unequal expected self-interested utilities, i.e. $E\left[X_{i}\right] \neq E\left[X_{j}\right]$. As in the outcome Fehr-Schmidt model, the utility reduction is larger when the inequality is disadvantageous than when it is advantageous. Now $\beta_{i}<1$ implies that the agent's expected all-inclusive utility is increasing in expected own self-interested utility. Non-negativity of the parameters implies that there is no ex-ante inequality seeking. The process model is able to accommodate experimental data that the outcome model cannot explain.

Both fairness concepts have been found to matter empirically and the Fehr-Schmidt type models successfully predict experimental data. Individual agents' utility functions can be assessed by observing their choices between actual allocations $\left(u_{1}, \ldots, u_{N}\right)$ for the outcome model and risky allocations $\left(u_{i}, X_{1}, \ldots, X_{N}\right)$ for the process model (Camerer and Fehr 2004; Rohde 2007). The self-interested vNM utilities can be assessed through standard utility measurement procedures (Keeney and Raiffa 1976; Wakker and Deneffe 1996; Abdellaoui 2000). Applying these tractable models therefore allows for a quantitative welfare evaluation under utilitarianism that is based on empirically relevant individual attitudes towards fairness.

Process and outcome fairness are closely related to a priori and a posteriori fairness. An agent with process fairness preferences takes the a priori view on fairness both before and after the resolution of uncertainty. An agent with outcome fairness preferences takes the a posteriori view at both points (Machina 1989; Trautmann 2007).

\section{Incorporating individual fairness in Diamond's and Broome's examples}

We discuss Diamond's (1967) and Broome's (1991) examples of social choices between random allocations when agents have Fehr-Schmidt preferences for either outcome or process fairness and $N=2$. We apply the two-stage procedure and assume that the utilities in Figs. 1 and 2 are self-interested vNM utilities that have been derived from self-interested choices between risky prospects over outcomes. These self-interested utilities enter the second-stage fairness models as inputs to obtain allinclusive utilities. For agent A and B's inequality aversion parameters in the outcome and process Fehr-Schmidt model we assume for simplicity that $\alpha_{A}=\alpha_{B}>0$ and $\beta_{A}=\beta_{B}>0$.

Example $1^{\prime}$ It has been shown above that without consideration of fairness the social planner's indifference between P and Q in Diamond's example can be unconvincing. Including fairness considerations at the individual level by applying the process FehrSchmidt model (3.5) to the allocation of self-interested utility specified in Fig. 1, we obtain the all-inclusive utilities given in Fig. 5.

Allocation procedure $\mathrm{Q}$ with expected welfare 1 is preferred over procedure $\mathrm{P}$ with expected welfare $1-\alpha-\beta$ by the social planner if subjects hold the process fairness view. The process model can immunize utilitarianism against the criticism raised by Diamond's example.

Applying the outcome Fehr-Schmidt model (3.4) to the example, we obtain the utilities given in Fig. 6. The utilitarian planner is indifferent between $\mathrm{P}$ and $\mathrm{Q}$ if subjects care about outcome fairness: under both allocation procedures they will always obtain 
Fig. 5 Process Fehr-Schmidt utilities in Diamond's example

Fig. 6 Outcome Fehr-Schmidt utilities in Diamond's example

Fig. 7 Outcome Fehr-Schmidt utilities in Broome's example
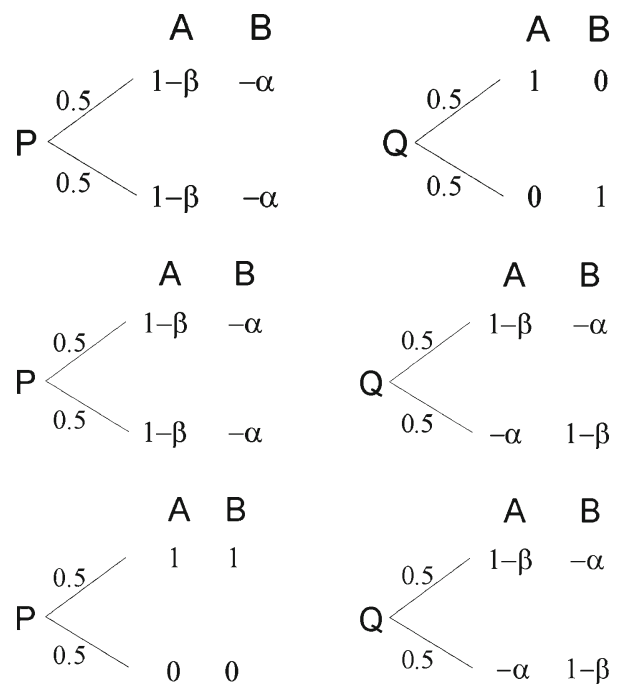

the same degree of outcome inequality. The example's criticism cannot be accommodated because it is always B who is worse off under process P, making the society's indifference unconvincing.

Example 2' In Broome's example indifference between procedures $\mathrm{P}$ and Q may not be convincing because Q always involves unequal self-interested vNM utilities while $\mathrm{P}$ gives both agents equal self-interested VNM utilities in each state. Applying the outcome Fehr-Schmidt model we obtain the all-inclusive utilities in Fig. 7.

Procedure $\mathrm{P}$ with expected welfare 1 is preferred over procedure $\mathrm{Q}$ that gives expected welfare $1-\alpha-\beta$. Including preferences for outcome fairness in the utilitarian welfare assessment accommodates the criticism raised by the example. If we apply the process Fehr-Schmidt model (3.5) to the allocations in Fig. 2 to obtain all-inclusive utilities, however, we obtain the same numbers as in Fig. 2: both subjects have equal expected self-interested utility under both procedures and inequality aversion terms drop out of the all-inclusive utility function. Both subjects, and therefore the social planner, are indifferent between allocation procedure $\mathrm{P}$ and Q. Utilitarianism with process fairness preferences does not account for the criticism raised by Broome's example.

The following table summarizes the appraisal of Harsanyi's utilitarianism in the two examples for the different fairness concepts.

\begin{tabular}{lll}
\hline & $\begin{array}{l}\text { Broome's } \\
\text { example }\end{array}$ & $\begin{array}{l}\text { Diamond's } \\
\text { example }\end{array}$ \\
\hline Self-interested & - & - \\
Outcome Fehr-Schmidt & + & - \\
Process Fehr-Schmidt & - & + \\
\hline +, criticism accommodated; - , criticism not accommodated
\end{tabular}


Applying self-interested individual utility in welfare assessments, the utilitarian prediction of the planner's preferences is not convincing for either example. Assuming that individuals' utilities include outcome fairness norms, utilitarianism can accommodate the criticism raised by Broome's example. Assuming process fairness, Diamond's criticism can be accommodated.

\section{Discussion}

By observing individual preference for process fairness or outcome fairness, the social planner can use the two-stage approach with the respective individual fairness model to determine the optimal allocation under utilitarianism. The social welfare evaluation considers fairness through all-inclusive utilities.

At the second stage, we used Fehr-Schmidt inequality aversion models to include individual fairness preferences. However, other fairness models can be incorporated in the two-stage approach if they appear more appropriate descriptions of individual preferences. Examples include the outcome and process fairness models of Bolton and Ockenfels (2000) and Bolton et al. (2005) that make different assumptions than Fehr-Schmidt in the $N$-person case, or the model of Charness and Rabin (2002) in which agents focus on efficiency and the worst-off person. Krawczyk (2007) provides a model of both outcome and process fairness. Gächter and Riedl (2006) and Traub etal. (2006) discuss more complex fairness norms at the individual level for which second-stage models could be developed and implemented through the twostage approach. Very different and possibly complex individual fairness notions can be accommodated by utilitarianism with all-inclusive utility.

Diecidue (2006) derives utilitarianism from a book-making argument under certainty. He assumes that the planner aggregates over many economic policies and that monetary equivalents exist for all consequences that the subjects face. Fairness can be included in this model by applying the second stage fairness model to individual monetary outcomes aggregated over all policies. These all-inclusive utilities can be used in the calculation of the aggregate welfare.

For some individual fairness second-stage models the utilitarian prediction may turn out to be identical to the prediction of some non-utilitarian social welfare model. If the two-stage model and the non-utilitarian model are empirically indistinguishable, the non-utilitarian model might be seen as a reduced form of the true two-stage utilitarian model (Machina 1984).

\section{Conclusion}

This article argues that fairness can be incorporated in Harsanyi's (1955) utilitarian welfare function at the individual level through all-inclusive utilities. A two-step procedure is proposed that uses tractable parametric models of individual fairness to operationalize all-inclusive utility. The approach retains Harsanyi's normative assumptions and relates fairness in welfare evaluation to observable individual preferences. Falsifiability and predictive power of utilitarianism are preserved. 
Fehr-Schmidt models of outcome and process fairness have been used to accommodate fairness-based criticisms of utilitarianism. The two-stage model is, however, a flexible tool to incorporate fairness in utilitarian social welfare. If tractable models of individual fairness are available which are empirically more successful or allow for more complex notions of fairness, then these can be used as well to incorporate fairness in utilitarianism.

Acknowledgements I am grateful to Peter P. Wakker and seminar participants at the Decision and Uncertainty Workshop 2006 in Paris and FUR XII in Rome for helpful comments.

Open Access This article is distributed under the terms of the Creative Commons Attribution Noncommercial License which permits any noncommercial use, distribution, and reproduction in any medium, provided the original author(s) and source are credited.

\section{References}

Abdellaoui, M. (2000). Parameter-free elicitation of utility and probability weighting functions. Management Science, 46(11), 1497-1512.

Binmore, K. (1994). Game theory and the social contract volume I: Playing fair. Cambridge, Mass: MIT Press.

Bolton, G. E., \& Ockenfels, A. (2000). ERC: A theory of equity, reciprocity, and competition. American Economic Review, 90(1), 166-193.

Bolton, G. E., Brandts, J., \& Ockenfels, A. (2005). Fair procedures: Evidence from games involving lotteries. Economic Journal, 115, 1054-1076.

Broome, J. (1991). Weighing goods—equality, uncertainty and time. Cambridge, Mass: Basil Blackwell.

Camerer, C. F., \& Fehr, E. (2004). Measuring social norms and preferences using experimental games: A guide for social scientists. In J. P. Henrich, R. Boyd, S. Bowles, C. F. Camerer, E. Fehr, \& H. Gintis (Eds.), Foundations of human sociology-economic experiments and ethnographic evidence from 15 small-scale societies (pp. 55-95). Oxford: Oxford University Press.

Charness, G., \& Rabin, M. (2002). Understanding social preferences with simple tests. Quarterly Journal of Economics, 117(3), 817-869.

Cox, J. C., \& Deck, C. A. (2005). On the nature of reciprocal motives. Economic Inquiry, 43(3), 623-635.

Diamond, P. A. (1967). Cardinal welfare, individualistic ethics, and interpersonal comparisons of utility: Comment. Journal of Political Economy, 75(5), 765- 766.

Diecidue, E. (2006). Deriving Harsanyi's utilitarianism from De Finetti's book-making argument. Theory and Decision, 61(4), 363-371.

Epstein, L. G., \& Segal, U. (1992). Quadratic social welfare functions. Journal of Political Economy, 100(4), 691-712.

Fehr, E., \& Schmidt, K. (1999). A theory of fairness, competition and cooperation. Quarterly Journal of Economics, 114(3), 817-868.

Fischer, J. A., \& Torgler, B. (2006). Does envy destroy social fundamentals? The impact of relative income position on social capital. UK: LSE.

Gächter, S., \& Riedl, A. (2006). Dividing justly in bargaining problems with claims: Normative judgments and actual negotiations. Social Choice and Welfare, 27(3), 571-594.

Grant, S., Kajii, A., Polak, B., \& Safra, Z. (2006). Generalized utilitarianism and Harsanyi's impartial observer theorem. UCLA.

Harsanyi, J. C. (1955). Cardinal welfare, individualistic ethics, and interpersonal comparisons of utility. Journal of Political Economy, 63(4), 309-321.

Karni, E. (1996). Social welfare functions and fairness. Social Choice and Welfare, 13(4), 487-496.

Karni, E., \& Safra, Z. (2002). Individual sense of justice: A utility representation. Econometrica, 70(1), 263-284.

Keeney, R. L., \& Raiffa, H. (1976). Decisions with multiple objectives. New York: Wiley.

Kelsey, D. (1994). Maxmin expected utility and weight of evidence. Oxford Economic Papers, 46(3), 425-444. 
Krawczyk, M. W. (2007). A model of procedural and distributive fairness. The Netherlands: University of Amsterdam.

Luce, R. D., \& Raiffa, H. (1957). Games and decisions. New York: Wiley.

Machina, M. J. (1984). Temporal risk and the nature of induced preferences. Journal of Economic Theory, 33(2), 199-231.

Machina, M. J. (1989). Dynamic consistency and non-expected utility models of choice under uncertainty. Journal of Economic Literature, 27(4), 1622-1668.

Rohde, K. I. M. (2007). A preference foundation for Fehr and Schmidt's model of inequality aversion. The Netherlands: Erasmus University Rotterdam.

Sugden, R. (2000). The motivating power of expectations. In J. Nida-Rümelin \& W. Spohn (Eds.), Rationality, rules, and structure (pp. 103-129), Dordrecht, the Netherlands: Kluwer Academic.

Traub, S., Seidl, C., \& Schmidt, U. (2006). An experimental study on individual choice, social welfare and social preference. Germany: University of Kiel.

Trautmann, S. T. (2007). Fehr-Schmidt process fairness and dynamic consistency. The Netherlands: Erasmus University Rotterdam.

Van Winden, F. (2007). Affect and fairness in economics. Social Justice Research, 20(1), 35-52.

Wakker, P. P., \& Deneffe, D. (1996). Eliciting von Neumann-Morgenstern utilities when probabilities are distorted or unknown. Management Science, 42(8), 1131-1150.

Wakker, P. P., \& Zank, H. (1999). State dependent expected utility for Savage's state space. Mathematics of Operations Research, 24(1), 8-34.

Zank, H. (2007). Social welfare functions with a reference income. Social Choice and Welfare, 28(4), $609-636$. 